\title{
The capacitated distribution and waste disposal problem
}

\author{
Jacqueline M. Bloemhof-Ruwaard ${ }^{a}$, Marc Salomon ${ }^{b, *}$, Luk N. Van Wassenhove ${ }^{c}$ \\ ${ }^{a}$ Landbouw Universiteit Wageningen, P.O. Box 9101, 6700 HB Wageningen, Netherlands \\ ${ }^{\mathrm{b}}$ Erasmus Universiteit Rotterdam, P.O. Box 1738, 3000 DR Rotterdam, Netherlands \\ ${ }^{\mathrm{c}}$ INSEAD, Boulevard de Constance, 77305 Fontainebleau Cedex, France
}

Received October 1993; revised February 1994

\begin{abstract}
We study the problem of the simultaneous design of a distribution network with plants and waste disposal units, and the coordination of product flows and waste flows within this network. The objective is to minimize the sum of fixed costs for opening plants and waste disposal units, and variable costs related to product and waste flows. The problem is complicated by (i) capacity constraints on plants and waste disposal units, (ii) service requirements (i.e. production must cover total demand) and (iii) waste, arising from production, to be disposed of at waste disposal units. We discuss alternative mathematical model formulations for the two-level distribution and waste disposal problem with capacity constraints. Lower bounding and upper bounding procedures are analyzed. The bounds are shown to be quite effective when embedded in a standard branch and bound algorithm. Finally, the results of a computational study are reported.
\end{abstract}

Keywords: Capacitated facility location; Mixed integer programming; Relaxations; Heuristics

\section{Introduction}

In the problem studied in this paper, we consider plants and waste disposal units (WDUs) to be located at selected sites. Product flows must be such that customer demand can be satisfied, and waste flows, arising from production, have to be disposed of at waste disposal units. In addition, there are restrictions on production capacity at the plants and disposal capacity at WDUs. The objective is to minimize the sum of fixed costs (arising from opening plants and WDUs), and variable costs (related to product and waste flows).

The problem is NP-hard, as it is a generaliza-

\footnotetext{
* Corresponding author.
}

tion of several other well-known NP-hard problems: (i) the one-level capacitated plant location problem (e.g. Francis et al., 1983; Cornuejols et al., 1991), (ii) the two-level capacitated facility location problem (e.g. Aardal, 1992), and (iii) the two-level uncapacitated distribution and waste disposal problem (Bloemhof-Ruwaard et al., 1994).

The problem described above is not only interesting from an academic point of view. It appears in practice when locating feedstock breeding farms and manure processing plants. When breeding pigs for the food processing industry, a huge quantity of manure is generated. This manure causes serious environmental problems, since it pollutes air, water, and soil. It contributes 
to acidification, leaching of minerals, unacceptable nitrate concentrations, etc. In our model the food processing industries can be considered as 'customers', the breeding farms as 'plants', and the manure processing plants as 'WDUs'.

The objectives of this paper are: (i) To outline alternative (mixed integer programming) formulations for the capacitated distribution and waste disposal problem (Section 2), (ii) to analyze the quality of several lower bounds that can be obtained for this problem (Section 3), and (iii) to develop heuristic procedures for obtaining feasible solutions (Section 4).

In order to test the effectiveness of the lower and upper bounding procedures, a computational study was carried out. Results of this study are reported in Section 5. Finally, in Section 6 we summarize our findings.

\section{Model formulations}

Mathematically, the capacitated distribution and waste disposal problem can be formulated as a mixed integer linear programming model. In what follows we present two alternative model formulations. The first model formulation is denoted by $M 1$.

$$
\begin{aligned}
z_{M 1}=\min & \sum_{i \in I} \sum_{j \in J} a_{i j} X_{i j}+\sum_{i \in I} f_{i} Y_{i} \\
& +\sum_{i \in I} \sum_{k \in K} a_{i k}^{w} X_{i k}^{w}+\sum_{k \in K} f_{k}^{w} Y_{k}^{w}
\end{aligned}
$$

subject to

$$
\begin{aligned}
& \sum_{i \in I} X_{i j}=d_{j}, j \in J, \\
& \sum_{j \in J} X_{i j} \leqslant s_{i} Y_{i}, i \in I, \\
& \sum_{j \in J} e_{i} X_{i j}=\sum_{k \in K} X_{i k}^{w}, i \in I, \\
& \sum_{i \in I} X_{i k}^{w} \leqslant s_{k}^{w} Y_{k}^{w}, k \in K, \\
& Y_{i}, Y_{k}^{w} \in\{0,1\}, i \in I, k \in K, \\
& X_{i j}, X_{i k}^{w} \geqslant 0, i \in I, j \in J, k \in K,
\end{aligned}
$$

where the following notation is used:

$I:=\{1, \ldots, m\}=$ Set of potential plant sites.

$J:=\{1, \ldots, n\}=$ Set of customers.

$K:=\{1, \ldots, p\}=$ Set of potential WDU sites.

The decision variables are:

$X_{i j}=$ Product flow from plant $i$ to customer $j$ (in units).

$X_{i k}^{w}=$ Waste flow from plant $i$ to WDU $k$ (in units).

$Y_{i}= \begin{cases}1 & \text { if WDU } i \text { is open } \\ 0 & \text { otherwise. }\end{cases}$

$Y_{k}^{w}= \begin{cases}1 & \text { if WDU } k \text { is open, } \\ 0 & \text { otherwise. }\end{cases}$

Parameters are:

$a_{i j}=$ Transportation costs (per unit of product flow) from plant $i$ to customer $j$.

$a_{i k}^{w}=$ Transportation costs (per unit of waste flow) from plant $i$ to WDU $k$.

$d_{j}=$ Demand from customer $j$.

$e_{i}=$ Waste fraction at plant $i$.

$f_{i}=$ Fixed cost for running plant $i$.

$f_{k}^{w}=$ Fixed cost for running WDU $k$.

$s_{i}=$ Capacity of plant $i$.

$s_{k}^{w}=$ Capacity of WDU $k$.

The objective (1) is to minimize the sum of total transportation costs and total fixed costs. Constraints (2) state that all customer demand must be satisfied. Constraints (3) and (5) are the capacity constraints for plants (WDUs), ensuring that no plant (WDU) produces (disposes) more than its capacity. Constraints (4) are the flow balancing constraints, stating that all waste resulting from production be disposed of at a WDU. Furthermore, (6) are the integrality constraints on the $Y$ and $Y^{w}$ variables, and (7) are the non-negativity constraints on the flows.

Valid inequalities can be derived to tighten the linear programming relaxation of $M 1$. Most of these valid inequalities are based on previous work for the single-level plant location problem. Valid-inequalities (8) and (9) are based on Davis and Ray (1969). Constraints (8) state that total flow between plant $i$ and customer $j$ can never 
exceed the minimum of customer $j$ 's demand and the capacity at plant $i$. Constraints (9) state that the total waste flow between plant $i$ and WDU $k$ can never be larger than the minimum of the capacity at WDU $k$, and the maximum waste generated at plant $i$,

$X_{i j} \leqslant \min \left\{d_{j}, s_{i}\right\} Y_{i}, i \in I, j \in J$,

$X_{i k}^{w} \leqslant \min \left\{w_{i}, s_{k}^{w}\right\} Y_{k}^{w}, i \in I, k \in K$,

where $\left(w_{i} \stackrel{\text { def }}{=} \sum_{j \in J} e_{i} d\right)$.

Valid-inequalities (10) and (11) below are used to set a lower bound on the number of plants and WDUs that have to be opened in order to obtain a feasible solution to $M 1$. In (10) (see Christofides and Beasley, 1983; Guignard and Opaswongkarn, 1990) the constant $\alpha$ is the smallest integer number of plants (with the largest capacities) that must be open in order to satisfy all the demand $\sum_{j \in J} d_{j}$ ). Similarly, constant $\beta$ in (11) is the smallest integer number of WDUs (with the largest capacities) that must be open in order to dispose the minimum waste flow $\left(\min _{i \in I} w_{i}\right)$ :

$$
\begin{aligned}
& \sum_{i \in I} Y_{i} \geqslant \alpha, \\
& \sum_{k \in K} Y_{k}^{w} \geqslant \beta .
\end{aligned}
$$

An alternative formulation of the distribution and waste disposal problem uses decision variables $X_{i j k}$ instead of decision variables $X_{i j}$ and $X_{i k}^{w}$. Here, $X_{i j k}$ denotes the fraction of customer $j$ 's demand, produced at plant $i$, with the waste disposed of at WDU $k$. Henceforth, we denote this alternative model formulation by $M 2$.

$$
\begin{aligned}
z_{M 2}=\min & \sum_{i \in I} \sum_{j \in J} \sum_{k \in K} c_{i j k} X_{i j k} \\
& +\sum_{i \in I} f_{i} Y_{i}+\sum_{k \in K} f_{k}^{w} Y_{k}^{w}
\end{aligned}
$$

subject to

$$
\sum_{i \in I} \sum_{k \in K} X_{i j k}=1, j \in J
$$

$$
\begin{aligned}
& \sum_{j \in J} \sum_{k \in K} d_{j} X_{i j k} \leqslant s_{i} Y_{i}, i \in I, \\
& \sum_{i \in I} \sum_{j \in J} d_{j} e_{i} X_{i j k} \leqslant s_{k}^{w} Y_{k}^{w}, k \in K, \\
& Y_{i}, Y_{k}^{w} \in\{0,1\}, i \in I, k \in K, \\
& 0 \leqslant X_{i j k} \leqslant 1, i \in I, j \in J, k \in K,
\end{aligned}
$$

where constant $c_{i j k}$ is the total variable cost for supplying customer $j$ 's demand from plant $i$, and disposing the waste at WDU $k$, i.e.

$c_{i j k}=\left(a_{i j}+a_{i k}^{w} e_{i}\right) d_{j}$.

Besides valid inequalities (10) and (11), the following inequalities are also 'valid' for $M 2$ :

$$
\begin{aligned}
& \sum_{k \in K} X_{i j k} \leqslant Y_{i}, i \in I, j \in J, \\
& \sum_{i \in I} X_{i j k} \leqslant Y_{k}^{w}, j \in J, k \in K .
\end{aligned}
$$

The above valid inequalities are introduced by Ro and Tcha (1984) in their paper on the uncapacitated two-level location problem. Inequalities (18) ensure that no flow exists between plant $i$, customer $j$, and any of the WDUs $k$, unless plant $i$ is open. Valid inequalities (19) are analogous to (18).

\section{Lower bounding procedures}

We discuss two types of lower bounding procedures to $M 1$ and $M 2$, i.e. (i) linear programming relaxations strengthened by valid inequalities (Section 3.1), and (ii) Lagrangean relaxations (Section 3.2).

\subsection{Linear programming relaxations}

Here we compare the relative quality of the linear programming relaxations of $M 1$ and $M 2$, when adding valid inequalities (8)-(11) to $M 1$ and (10), (11), (18), and (19) to $M 2$. For this comparison we introduce the following notation:

- Constraint set (8) and (9) is denoted by $A$,

- Constraint set (10) and (11) is denoted by $B$;

- Constraint set (18) and (19) is denoted by $C$. 
For example, $Z_{\mathrm{LP}\left(M 1^{A B}\right)}$ is the optimal solution value of the LP-relaxation to $M 1$, when valid inequalities $\boldsymbol{A}$ and $\boldsymbol{B}$ are added to it.

We first prove the following lemmas:

Lemma 1. $Z_{\mathrm{LP}(M 1)}=Z_{\mathrm{LP}(M 2)}$.

Proof. The following equivalence relations hold by definition:

(a) $X_{i j}=\sum_{k \in K} d_{j} \times X_{i j k}$.

(b) $X_{i k}^{w}=\sum_{j \in J} e_{i} \times d_{j} \times X_{i j k}$.

(c) $\left(a_{i j}+a_{i k}^{w} \times e_{i}\right) \times d_{j}=c_{i j k}$.

Given these equivalence relations, we prove that LP (M1) is equivalent to LP (M2). The objectives (1) and (12) in both formulations are equivalent:

$$
\begin{aligned}
& \sum_{i \in I} \sum_{j \in J} \sum_{k \in K} c_{i j k} X_{i j k}+\sum_{i \in I} f_{i} Y_{i}+\sum_{k \in K} f_{k}^{w} Y_{k}^{w} \\
& \stackrel{\text { (c) }}{=} \sum_{i \in I} \sum_{j \in J} \sum_{k \in K}\left(a_{i j}+a_{i k}^{w} \times e_{i}\right) \times d_{j} X_{i j k} \\
& \quad+\sum_{i \in I} f_{i} Y_{i}+\sum_{k \in K} f_{k}^{w} Y_{k}^{w} \\
& =\sum_{i \in I} \sum_{j \in J} a_{i j} \sum_{k \in K} d_{j} X_{i j k} \\
& \quad+\sum_{i \in I} \sum_{k \in K} a_{i k}^{w} \sum_{j \in J} e_{i} \times d_{j} \times X_{i j k} \\
& \quad+\sum_{i \in I} f_{i} Y_{i}+\sum_{k \in K} f_{k}^{w} Y_{k}^{w} \\
& \stackrel{\text { (a),(b) }}{=} \sum_{i \in I} \sum_{j \in J} a_{i j} X_{i j}+\sum_{i \in I} \sum_{k \in K} a_{i k}^{w} X_{i k}^{w} \\
& \quad+\sum_{i \in I} f_{i} Y_{i}+\sum_{k \in K} f_{k}^{w} Y_{k}^{w} .
\end{aligned}
$$

Also, demand constraints (2) and (13) are equivalent, since for each $j=1, \ldots, n$,

$$
\sum_{i \in I} \sum_{k \in K} X_{i j k}=1 \Leftrightarrow d_{j} \sum_{i \in I} \sum_{k \in K} X_{i j k}=d_{j}
$$

$$
\stackrel{\text { (a) }}{\Leftrightarrow} \sum_{i \in I} X_{i j}=d_{j} \text {. }
$$

Furthermore, flow balancing constraints (4) are automatically satisfied in $M 2$, since for each $i=$ $1, \ldots, m$,

$$
\begin{aligned}
& \sum_{j \in J} e_{i} X_{i j} \stackrel{(\mathrm{a})}{=} \sum_{j \in J} e_{i} \sum_{k \in K} d_{j} X_{i j k} \\
& =\sum_{j \in J} \sum_{j \in J} e_{i} d_{j} X_{i j k}=\sum_{k \in K} X_{i k}^{w} .
\end{aligned}
$$

By the definition of (a) it follows that (3) is equivalent to (14), and by the definition of (b) it follows that (5) and (15) are equivalent.

Lemma 2. $Z_{\mathrm{LP}\left(M 1^{A B}\right)} \leqslant Z_{\mathrm{LP}\left(M 2^{B C}\right)}$.

Proof. In the Appendix we show that there exists an instance for which the solution to $\operatorname{LP}\left(M 2^{A}\right)$ is not feasible in $\operatorname{LP}\left(M 2^{C}\right)$. Next we prove that every feasible solution to $\operatorname{LP}\left(M 2^{B C}\right)$ is feasible to $\mathrm{LP}\left(M 1^{A B}\right)$.

To do so, consider an arbitrary feasible solution to $\operatorname{LP}\left(M 2^{B C}\right)$. We know from Lemma 1 that $Z_{\mathrm{LP}\left(M 1^{B}\right)}=Z_{\mathrm{LP}\left(M 2^{B}\right)}$. It remains to be shown that (8) and (9) are satisfied by the solution to $\mathrm{LP}\left(M 2^{B C}\right)$. Constraints (18) can be rewritten as

$$
\begin{aligned}
& \sum_{k \in K} X_{i j k} \leqslant Y_{i} \\
& \Leftrightarrow \sum_{k \in K} d_{j} X_{i j k} \leqslant d_{j} Y_{i} \stackrel{(\mathrm{a})}{\Leftrightarrow} X_{i j} \leqslant d_{j} Y_{i} .
\end{aligned}
$$

Two cases may occur now:

Case 1: $\min \left(d_{j}, s_{i}\right)=s_{i}$. Then (8) reduces to $X_{i j} \leqslant s_{i} Y_{i}$. Consequently, (8) is dominated by (3), and thus (8) is satisfied,

Case 2: $\min \left(d_{j}, s_{i}\right)=\mathrm{d}_{j}$. Then $X_{i j} \leqslant d_{j} Y_{i}$ is equivalent to (8).

Constraints (19) can be rewritten as

$$
\sum_{i \in I} X_{i j k} \leqslant Y_{k}^{w} \Leftrightarrow \sum_{j \in J} d_{j} \sum_{i \in I} X_{i j k} \leqslant \sum_{j \in J} d_{j} Y_{k}^{w}
$$

Consider now one single plant $i^{*}$. Then

$$
\begin{aligned}
e_{i} & \sum_{j \in J} d_{j} \sum_{i \in I} X_{i j k} \leqslant e_{i} \cdot \sum_{j \in J} d_{j} Y_{k}^{w} \\
& \Leftrightarrow \sum_{j \in J} d_{j} e_{i} X_{i * j k}+\sum_{j \in J} d_{j} e_{i} \cdot \sum_{i \neq i^{*}} X_{i j k}
\end{aligned}
$$




$$
\begin{aligned}
& \leqslant \sum_{j \in J} d_{j} e_{i} \cdot Y_{k}^{w} \stackrel{\text { (b) }}{\Leftrightarrow} X_{i^{*} k}^{w}+\sum_{j \in J} d_{j} e_{i} \cdot \sum_{i \neq i^{*}} X_{i j k} \\
& \leqslant w_{i^{*}} Y_{k}^{w} \Rightarrow X_{i^{*} k}^{w} \leqslant w_{i^{*}} Y_{k}^{w} .
\end{aligned}
$$

The above results hold for all $i^{*} \in I$. Again, two cases may occur now:

Case 1: $\min \left(w_{i}, s_{k}^{w}\right)=s_{k}^{w}$. Then (9) reduces to $X_{i k}^{w} \leqslant s_{k}^{w} Y_{k}^{w}$. Consequently, (9) is dominated by (5), and thus (9) is satisfied.

Case 2: $\min \left(w_{i}, s_{k}^{w}\right)=w_{i}$. Then $X_{i k}^{w} \leqslant w_{i} Y_{k}^{w}$ is equivalent to (9).

Lemma 3. No dominance relations exist between $Z_{\mathrm{LP}\left(M 1^{A}\right)}$ and $Z_{\mathrm{LP}\left(M 1^{B}\right) \text {, between } Z_{\mathrm{LP}\left(M 2^{B}\right)} \text { and }}$ $Z_{\mathrm{LP}\left(M 2^{C}\right)}, Z_{\mathrm{LP}\left(M 1^{A}\right)}$ and $Z_{\mathrm{LP}\left(M 2^{B}\right)}$, and between $Z_{\mathrm{LP}\left(M 1^{B}\right)}$ and $Z_{\mathrm{LP}\left(M 2^{C}\right)}$.

Proof. Consider problem instance I with two customers, two plants, and two WDUs. Customer demands are $d_{1}=2$ and $d_{2}=3$. Transportation costs are $a_{21}=3$ and $a_{12}^{w}=3$. All other transportation costs are zero. Fixed costs for opening plants are $f_{1}=2$ and $f_{2}=3$, and for the WDUs $f_{1}^{w}=2$ and $f_{2}^{w}=3$. Capacity of the plants are $s_{1}=3$ and $s_{2}=5$, and for the WDUs $s_{1}^{w}=3$ and $s_{2}^{w}=5$. Waste percentages are $e_{i}=1$ for $i=1,2$. Problem instance II is identical to problem instance I, except that $s_{2}=4$ and $s_{2}^{w}=4$. The optimal solutions to these instances are presented in Table 1.

The results for instances I and II indeed show that no dominance relations exist between $Z_{\mathrm{LP}\left(M 1^{A}\right)}$ and $Z_{\mathrm{LP}\left(M 1^{B}\right)}$, between $Z_{\mathrm{LP}\left(M 2^{B}\right)}$ and between $Z_{\mathrm{LP}\left(M 1^{A}\right)}$ and $Z_{\mathrm{LP}\left(M 2^{B}\right)}$, and between $Z_{\mathrm{LP}\left(M 1^{B}\right)}$ and $Z_{\mathrm{LP}\left(M 2^{C}\right)}$.

\subsection{Lagrangean relaxation}

As an alternative to the linear programming based lower bounding procedures, we also obtain

Table 1

Solutions to instances I and II

\begin{tabular}{lll}
\hline Objective function & Instance I & Instance II \\
\hline$Z_{\mathrm{LP}\left(M 1^{A}\right)}$ & $7 \frac{1}{5}$ & $7 \frac{1}{2}$ \\
$Z_{\mathrm{LP}\left(M 1^{B}\right)}, Z_{\mathrm{LP}\left(M 2^{B}\right)}$ & $6 \frac{4}{15}$ & 10 \\
$Z_{\mathrm{LP}\left(M 2^{C}\right)}$ & 8 & 8 \\
\hline
\end{tabular}

lower bounds from Lagrangean relaxation of the flow-balancing constraints (4) of model formulation $M 1^{A}$. We denote the resulting problem by $\operatorname{LR}\left(M 1^{A}\right)$. The latter problem decomposes into the subproblems $\operatorname{LR}_{1}\left(M 1^{A}\right)$ and $\operatorname{LR}_{2}\left(M 1^{A}\right)$ as stated below:

$$
\begin{aligned}
& z_{\mathrm{LR}_{1}\left(M 1^{A}\right)}(\lambda) \\
& \quad=\min \sum_{i \in I} \sum_{j \in J}\left(a_{i j}+\lambda_{i} e_{i}\right) X_{i j}+\sum_{i \in I} f_{i} Y_{i}
\end{aligned}
$$

subject to

(2), (3), and (8),

$Y_{i} \in\{0,1\}, i \in I$,

$X_{i j} \geqslant 0, i \in I, j \in J$.

$$
\begin{aligned}
& z_{\mathrm{LR}_{2}\left(M 1^{A}\right)}(\lambda) \\
& \quad=\min \sum_{i \in I} \sum_{k \in K}\left(a_{i k}^{w}-\lambda_{i}\right) X_{i k}^{w}+\sum_{k \in K} f_{k}^{w} Y_{k}^{w}
\end{aligned}
$$

subject to

(5), and (9)

$Y_{k}^{w} \in\{0,1\}, k \in K$,

$X_{i k}^{w} \geqslant 0, i \in I, k \in K$,

where $\lambda=\left(\lambda_{1}, \ldots, \lambda_{m}\right)$ is the set of Lagrangean multipliers corresponding to (4). Note that $z_{\mathrm{LR}\left(m 1^{A}\right)}(\lambda)=z_{\mathrm{LR}_{1}\left(M 1^{A}\right)}(\lambda)+z_{\mathrm{LR}_{2}\left(M 1^{A}\right)}(\lambda)$.

Problem $\mathrm{LR}_{1}\left(M 1^{A}\right)$ is a single level capacitated plant location problem, which can be solved to optimality using a special purpose code like the one developed by Ryu and Guignard (1992). Due to the block structure of the constraint matrix, problem $\operatorname{LR}_{2}\left(M 1^{A}\right)$ decomposes into $p$ independent subproblems $\operatorname{LR}_{2}\left(M 1^{A}\right)_{k}(k=1, \ldots, p)$. The solution procedure to $\operatorname{LR}_{2}\left(M 1^{A}\right)_{k}$ is as follows:

\section{Solution procedure to $\operatorname{LR}_{2}\left(M 1^{A}\right)_{k}$.}

Step 1. Fix $Y_{k}^{w}=1$. Let $I_{\mathrm{k}}$ be the set of plants $i$ for which

$a_{i k}^{w}-\lambda_{i}<0$.

Next we formulate the following bounded continuous knapsack problem (BCKP):

(BCKP)

$\max \sum_{i \in I_{k}}\left(\lambda_{i}-a_{i k}^{w}\right) X_{i k}^{w}$ 


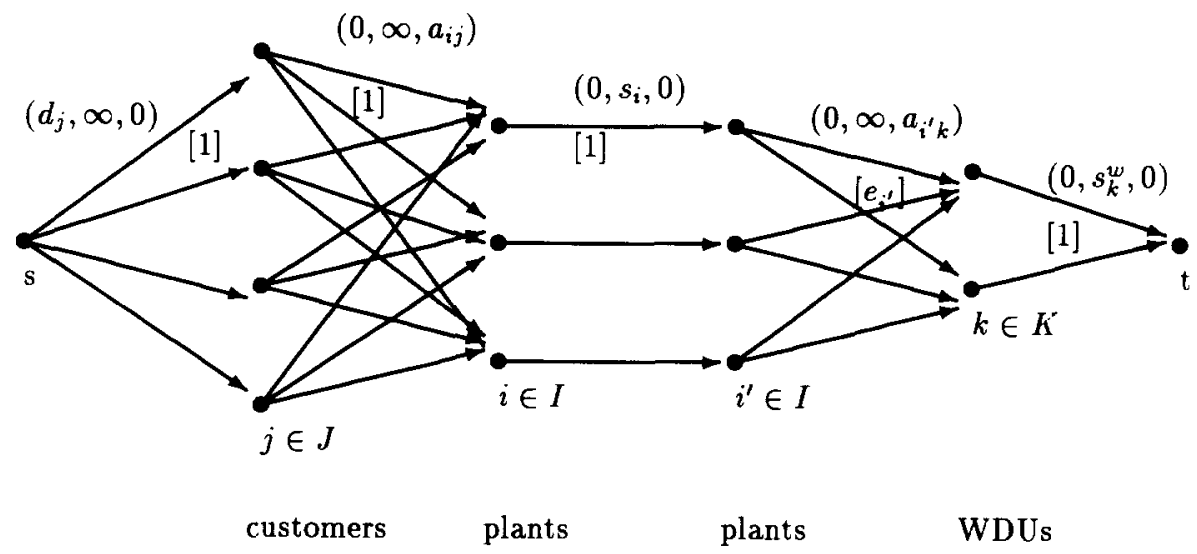

Fig. 1. The generalized minimum cost flow problem with losses. Here, the vector of lower bounds, upper bounds and costs corresponding to each arc from node $p$ to node $q$ is denoted by $\left(l_{p q}, u_{p q}, c_{p q}\right)$. The flow-intensity corresponding to the flow between node $p$ and $q$ is represented by $\left[r_{p q}\right]$. Note that a fraction of $1-r_{p q}$ of flow is lost between node $p$ and node $q$. Finally, node $s$ is the super source, node $t$ is the super sink, and nodes $i^{\prime}$ are introduced in order to represent capacity restrictions on plants.

subject to

$\sum_{i \in I_{k}} X_{i k}^{w} \leqslant s_{k}^{w}$

$0 \leqslant X_{i k}^{w} \leqslant \min \left\{s_{k}^{w}, w_{i}\right\}, i \in I_{k}$.

In order to solve BCKP we apply the Q( $\left(I_{k}|\log | I_{k} \mid\right)$ solution procedure of Martello and Toth $(1990, \mathrm{p} .84)$. This procedure yields solutions $\tilde{X}_{i k}^{w}$ (to be used in Step 2 below).

Step 2. The optimal solution to $\operatorname{LR}_{2}\left(M 1^{A}\right)_{k}$ is obtained using the following rule:

If

$f_{k}^{w}+\sum_{i \in I_{k}}\left(a_{i k}^{w}-\lambda_{1}\right) \tilde{X}_{i k}^{w}<0$,

then

$Y_{k}^{w}=1, X_{i k}^{w}=\tilde{X}_{i k}^{w}$ for $i \in I_{k}$, and

$X_{i k}^{w}=0$ for $i \notin I_{k}$.

Otherwise $Y_{k}^{w}=0$ and $X_{i k}^{w}=0$ for all $i \in I$. The costs corresponding to this solution are $Z_{\mathrm{LR}_{2}\left(M 1^{A}\right)_{k}}(\lambda)$.

Note that

$Z_{\mathrm{LR}_{2}\left(M 1^{A}\right)}(\lambda)=\sum_{k=1}^{p} Z_{\mathrm{LR}_{2}\left(M 1^{A}\right)_{k}}(\lambda)$.
The complete Lagrangean relaxation procedure can now be summarized as follows:

\section{Lagrangean relaxation procedure:}

Initialization: Set dual multipliers $\lambda=\left(\lambda_{1}, \ldots, \lambda_{m}\right)$ equal to zero.

Step 1. Solve $\operatorname{LR}_{1}\left(M 1^{A}\right)$ and $\operatorname{LR}_{2}\left(M 1^{A}\right)$ using the procedures described above.

Step 2. If convergence conditions are satisfied, then STOP, otherwise update dual multipliers $\lambda$ using subgradient optimization and return to Step 1.

Lemma 4. $Z_{\mathrm{LP}\left(M 1^{A}\right)} \leqslant \max Z_{\mathrm{LR}\left(M 1^{A}\right)}(\lambda)$.

Proof. This follows directly from Geoffrion (1974), since the integrality property does not apply LPrelaxation of $\operatorname{LR}_{1}\left(M 1^{A}\right)$.

The above lemma states that the Lagrangean lower bound may dominate the bounds resulting from the LP-relaxation of $M_{1}$. Also, as a consequence of Lemma 1, the Lagrangean lower bound may dominate $z_{\mathrm{LP}(M 2)}$. However, a number of computational experiments have shown that the procedure is largely outperformed by the linear programming based procedures in terms of required CPU time. The considerable effort re- 
quired to compute $Z_{\mathrm{LR}_{1}\left(M_{1} 1^{A}\right)}(\lambda)$ at each iteration of the subgradient optimization procedure does not seem to be compensated by superior values for the bounds. Therefore, in our computational study we refrain from further consideration of the Lagrangean relaxation technique.

Remark. As an alternative to the Lagrangean relaxation of constraints (4) we could also have experimented with Lagrangean relaxation of capacity constraints (3) and (5) of $M 1$, or (14) and (15) of $M 2$. Note that, in both cases, the resulting problem is a two-level uncapacitated distribution and waste disposal problem. This problem is still hard to solve to optimality (Bloemhof-Ruwaard et al., 1994). Therefore, we do not consider these relaxations in our study.

\section{Upper bounding procedures}

In what follows we discuss two alternative upper bounding procedures: (i) a linear programming round-off heuristic, and (ii) a sequential capacitated facility location heuristic.

\subsection{Linear programming round-off heuristic}

The linear programming round-off heuristic (LPRH) can be described as follows. Given the solution to the linear programs $\mathrm{LP}(M 1)$ or $\mathrm{LP}(M 2)$ we fix the binary variables $Y_{i}$ and $Y_{k}^{w}$ using a round-off strategy. The remaining problem is a minimum cost network flow problem with losses (MCNFPL) (see Fig. 1).

The Linear programming round-off heuristics can now be stated more formally as follows:

\section{Linear programming round-off heuristics:}

Step 1. For heuristic LPRH1 (LPRH2) solve $L P(M I) L P(M 2)$ ), yielding the (possibly fractional) solution values $\left(\tilde{Y}_{i}^{\prime}, \tilde{Y}_{k}^{w}\right)$ for the binary variables.

Step 2. Fix $\left.Y_{i}=1\left(Y_{k}^{w}=1\right)\right)$ when $\tilde{Y}_{i}>0\left(\tilde{Y}_{k}^{w}>\right.$ $0)$. Otherwise, set $Y_{i}=0\left(Y_{k}^{w}=0\right)$.

Step 3. Given the open plants and WDUs obtained from Step 2, design the corresponding minimum cost flow network with losses. Solve
MCNFPL using an appropriate network simplex procedure or a special purpose code (in our implementation we applied the simplex procedure available in IBM's library OSL).

Step 4. Compute the values $Z_{\mathrm{LPRH}_{1}}\left(Z_{\mathrm{LPRH}_{2}}\right)$ by adding fixed costs for opening plants and WDUs to $Z_{\text {MCNFPL }}$.

\subsection{Sequential capacitated facility location heuris- tic}

The sequential capacitated facility location heuristic (SCFLH) is based on the observation that a feasible solution to the Capacitated Distribution and Waste Disposal Problem can be constructed in two stages. In the first stage a single level capacitated facility location problem (between plants and customers) is solved. Given the solution to this problem, the amount of waste at each plant is computed. In the second stage of the heuristic again a single level capacitated facility location problem (between plants and WDUs) is solved, taking into account the amount of waste computed at the first stage. This two stage decomposition procedure is in fact similar to the decomposition that is applied within the Lagrangean lower bounding procedure. Unfortunately, the latter procedure does not yield feasible solutions automatically, as $\operatorname{LR}_{2}\left(M 1^{A}\right)$ does not explicitly take into account the amount of waste generated at each plant. However, a feasible solution is constructed by replacing $\operatorname{LR}_{2}\left(M 1^{A}\right)$ by subproblem $\operatorname{LR}_{2}^{E}\left(M 1^{A}\right)$ as defined below,

$$
\begin{aligned}
\left(\operatorname{LR}_{2}^{E}\left(\mathrm{M}^{A}\right)\right) & \\
z_{\operatorname{LR}_{2}^{E}\left(M 1^{A}\right)}(\lambda)= & \min \sum_{i \in I} \sum_{k \in K}\left(a_{i k}^{w}-\lambda_{i}\right) X_{i k}^{w} \\
& +\sum_{k \in K} f_{k}^{w} Y_{k}^{w}
\end{aligned}
$$

subject to

$$
\begin{aligned}
& \sum_{k \in K} X_{i k}^{w}=E_{i}, i \in I, \\
& \sum_{i \in I} X_{i k}^{w} \leqslant s_{k}^{w} Y_{k}^{w}, k \in K, \\
& X_{i k}^{w} \leqslant \min \left\{s_{k}^{w}, w_{i}\right\} Y_{k}^{w}, i \in I, k \in K,
\end{aligned}
$$


Table 2

Test problem characteristics of Set I

\begin{tabular}{rlrrrrrr}
\hline$\#$ & $f_{i}$ & \multicolumn{1}{c}{$s_{i}$} & \multicolumn{1}{c}{$f_{k}^{w}$} & \multicolumn{1}{c}{$s_{k}^{w}$} & $e_{i}$ & $\rho$ & $\rho^{w}$ \\
\hline 1 & 7500 & 5000 & 2500 & 1000 & $U[0.1 ; 0.2]$ & 0.61 & 0.65 \\
2 & 7500 & 5000 & 5000 & 3000 & $U[0.1 ; 0.2]$ & 0.61 & 0.22 \\
3 & 7500 & 5000 & 15000 & 11500 & $U[0.1 ; 0.2]$ & 0.61 & 0.06 \\
4 & 7500 & 5000 & 8000 & 5000 & $U[0.5 ; 1.0]$ & 0.61 & 0.65 \\
5 & 7500 & 5000 & 12500 & 15000 & $U[0.5 ; 1.0]$ & 0.61 & 0.22 \\
6 & 7500 & 5000 & 25000 & 57500 & $U[0.5 ; 1.0]$ & 0.61 & 0.06 \\
7 & 10000 & 10000 & 2500 & 1000 & $U[0.1 ; 0.2]$ & 0.30 & 0.65 \\
8 & 10000 & 10000 & 5000 & 3000 & $U[0.1 ; 0.2]$ & 0.30 & 0.22 \\
9 & 10000 & 10000 & 15000 & 11500 & $U[0.1 ; 0.2]$ & 0.30 & 0.06 \\
10 & 10000 & 10000 & 8000 & 5000 & $U[0.5 ; 1.0]$ & 0.30 & 0.65 \\
11 & 10000 & 10000 & 12500 & 15000 & $U[0.5 ; 1.0]$ & 0.30 & 0.22 \\
12 & 10000 & 10000 & 25000 & 57500 & $U[0.5 ; 1.0]$ & 0.30 & 0.06 \\
13 & 12500 & 15000 & 2500 & 1000 & $U[0.1 ; 0.2]$ & 0.20 & 0.65 \\
14 & 12500 & 15000 & 5000 & 3000 & $U[0.1 ; 0.2]$ & 0.20 & 0.22 \\
15 & 12500 & 15000 & 15000 & 11500 & $U[0.1 ; 0.2]$ & 0.20 & 0.06 \\
16 & 12500 & 15000 & 8000 & 5000 & $U[0.5 ; 1.0]$ & 0.20 & 0.65 \\
17 & 12500 & 15000 & 12500 & 15000 & $U[0.5 ; 1.0]$ & 0.20 & 0.22 \\
18 & 12500 & 15000 & 25000 & 57500 & $U[0.5 ; 1.0]$ & 0.20 & 0.06
\end{tabular}

$Y_{k}^{w} \in\{0,1\}, k \in K$,

$X_{i k}^{w} \geqslant 0, i \in I, k \in K$,

where the total amount of waste at plant $i$ is computed as

$E_{i} \stackrel{\text { def }}{=} \sum_{j \in J} e_{i} X_{i j}$

The values for the decision variables $X_{i j}$ are obtained from $\operatorname{LR}_{1}\left(M 1^{A}\right)$. In principle, for each set of Lagrangean multipliers a (new) upper bound can be obtained. However, because of the considerable computational requirements of an iterative procedure we limit our computational experiments to a iteration (with $\lambda=0$ ). Heuristic SCFLH is summarized as follows:

\section{Heuristic SCFLH.}

Step 1. Use the solution to $\operatorname{LR}_{1}\left(M 1^{A}\right)$ with $\lambda=0$ to compute $E_{i}$ for all $i \in I$. Solve $\operatorname{LR}_{2}^{E}\left(M 1^{A}\right)$ using the special purpose code by Ryu and Guignard (1992).

Step 2. Compute the value of the upper bound $Z_{\text {SCFLH }}$ corresponding to the solution of the heuristic.

\section{Computational study}

In this section we explain how we generated the test problems for the computational study (Section 5.1), and we discuss the computational results (Section 5.2).

\subsection{Problem generation}

We generated two sets of test problems. ${ }^{1}$ The sets differ with respect to the generation of fixed costs, capacities, and the ratio between available capacity and required capacity (capacity utilization) for plants and WDUs. Here, capacity utilization of plants is defined as

$\rho:=\left(\sum_{j} d_{j}\right) /\left(\sum_{i} s_{i}\right)$

and capacity utilization of WDUs is defined as

$\rho^{w}=\left(\bar{e} \sum_{j} d_{j}\right) /\left(\sum_{k} s_{k}^{w}\right)$

\footnotetext{
${ }^{1}$ Test problems are available from the authors upon request.
} 
where $\bar{e}$ is the average waste fraction, defined below. We now describe the specific characteristics of both problem sets (Set I and Set II).

\section{Set I}

Set I consists of 18 problem instances with 16 plants, 10 WDUs, and 30 customers. For each problem instance, fixed costs $\left(f_{i}\right.$ and $\left.f_{k}^{w}\right)$, and capacities $\left(s_{i}\right.$ and $\left.s_{k}^{w}\right)$ are varied as shown in Table 2. Variable transportation costs $\left(a_{i j}\right.$, and $\left.a_{i k}^{w}\right)$ and demands $\left(d_{j}\right)$ are taken from the single level problem instances used in a computational study by Khumawala (1974). Waste fractions $\left(e_{i}\right)$ are randomly generated from a uniform $U\left[e^{\mathrm{min}}\right.$; $\left.e^{\max }\right]$ distribution, where for each WDU, $e^{\min }$ is the minimum waste fraction and $e^{\max }$ is the maximum waste fraction. Note that

$\bar{e}=\frac{1}{2}\left(e^{\min }+e^{\max }\right)$.

In order to analyze the influence of capacity utilization on the performance of the lower bounding and upper bounding procedures, capacities of plants and WDUs and waste fractions of plants are chosen such that (expected) capacity utilization at plants (WDUs) is either HIGH with $\rho=0.61 \quad\left(\rho^{w}=0.65\right)$, MEDIUM with $\rho=0.30$ $\left(\rho^{w}=0.22\right)$, or LOW with $\rho=0.20\left(\rho^{w}=0.06\right)$.

Domschke and Drexl (1985) and Cornuejols et al. (1991) observe that the performance of their heuristics for the capacitated plant location problem is rather sensitive to high variabilities in fixed costs and capacity (utilization). In order to investigate whether this observation also applies to our lower bounding and upper bounding procedures for the problem studied here, we generated problem Set II.

\section{Set II}

Set II consists of 20 problem instances with the same number of plants, WDUs and customers as the problems generated by Khumawala (see also Set I). Capacities and fixed costs are generated as suggested by Cornuejols et al. (1991):

$$
\begin{aligned}
& s_{i}=U[3,500 ; 56,000], \\
& s_{k}^{w}=U[2,500 ; 40,000], \\
& f_{i}=U[0 ; 90]+U[100 ; 110] \sqrt{s_{i}}, \\
& f_{k}^{w}=U[0 ; 90]+U[100 ; 110] \sqrt{s_{k}^{w}} .
\end{aligned}
$$

\begin{tabular}{|c|c|c|c|c|c|c|c|c|}
\hline \multirow[b]{2}{*}{ Relaxation } & \multicolumn{4}{|c|}{ Quality of the solutions } & \multicolumn{4}{|c|}{ CPU-time in seconds } \\
\hline & $\overline{\Delta Z^{\mathrm{LB}}}$ & $\Delta \mathcal{Z}^{\mathrm{LB}, \min }$ & $\Delta Z^{\mathrm{LB}, \max }$ & $\sigma^{2}\left(\Delta Z^{\mathrm{LB}}\right)$ & $\overline{\mathrm{CPU}}{ }^{\mathrm{LB}}$ & $\mathrm{CPU}^{\mathrm{LB}, \min }$ & $\mathrm{CPU}^{\mathrm{LB}, \max }$ & $\sigma^{2}\left(\mathrm{CPU}^{\mathrm{LB}}\right)$ \\
\hline \multicolumn{9}{|l|}{ Set I: } \\
\hline $\mathbf{L P}(M 1)$ & 7.95 & 1.19 & 15.58 & 12.93 & 0.39 & 0.27 & 0.54 & 0.01 \\
\hline $\mathrm{LP}\left(M 1^{B}\right)$ & 7.95 & 1.19 & 15.58 & 12.93 & 0.39 & 0.25 & 0.58 & 0.01 \\
\hline $\operatorname{LP}\left(M 1^{A}\right)$ & 2.92 & 0.17 & 8.76 & 6.45 & 2.19 & 1.03 & 3.73 & 0.53 \\
\hline $\operatorname{LP}\left(M 1^{A, B}\right)$ & 2.92 & 0.17 & 8.76 & 6.45 & 2.18 & 1.05 & 3.70 & 0.53 \\
\hline $\mathrm{LP}(M 2)$ & 7.95 & 1.19 & 15.58 & 12.93 & 2.64 & 1.40 & 4.05 & 0.45 \\
\hline $\mathrm{LP}\left(M 2^{B}\right)$ & 7.95 & 1.1 .9 & 15.58 & 12.93 & 2.64 & 1.41 & 4.09 & 0.50 \\
\hline $\mathrm{LP}(M 2)$ & 0.36 & 0.00 & 1.48 & 0.14 & 120.56 & 69.06 & 189.01 & 1286.73 \\
\hline $\mathrm{LP}\left(M 2^{B, C}\right)$ & 0.36 & 0.00 & 1.48 & 0.14 & 120.48 & 68.75 & 189.12 & 1287.84 \\
\hline \multicolumn{9}{|l|}{ Set II: } \\
\hline $\mathbf{L P}(M 1)$ & 12.16 & 6.46 & 15.14 & 4.28 & 0.35 & 0.25 & 0.45 & 0.00 \\
\hline $\mathrm{LP}\left(M 1^{B}\right)$ & 12.16 & 6.46 & 15.14 & 4.28 & 0.34 & 0.26 & 0.44 & 0.00 \\
\hline $\operatorname{LP}\left(M 1^{A}\right)$ & 3.74 & 1.28 & 6.37 & 1.69 & 2.03 & 0.83 & 4.65 & 1.25 \\
\hline $\mathrm{LP}\left(M 1^{A, B}\right)$ & 3.74 & 1.28 & 6.37 & 1.69 & 2.04 & 0.83 & 4.58 & 1.24 \\
\hline $\mathrm{LP}(M 2)$ & 12.16 & 6.46 & 15.14 & 4.28 & 2.03 & 1.39 & 2.75 & 0.10 \\
\hline $\mathrm{LP}\left(M 2^{B}\right)$ & 12.16 & 6.46 & 15.14 & 4.28 & 1.99 & 1.01 & 2.71 & 0.14 \\
\hline $\mathrm{LP}\left(M 2^{C}\right)$ & 0.23 & 0.00 & 1.94 & 0.22 & 193.34 & 161.87 & 270.67 & 819.50 \\
\hline $\operatorname{LP}\left(M 2^{B, C}\right)$ & 0.23 & 0.00 & 1.94 & 0.22 & 193.30 & 162.27 & 270.86 & 830.50 \\
\hline
\end{tabular}

Table 3a

Summary of results for lower bounding procedures 
Table 3b

Summary of results for yoowe bounding procedures

\begin{tabular}{|c|c|c|c|c|c|c|c|c|}
\hline \multirow[b]{2}{*}{ Heuristic } & \multicolumn{4}{|c|}{ Quality of the solutions } & \multicolumn{4}{|c|}{ CPU-time in seconds } \\
\hline & $\overline{\Delta Z^{\mathrm{UB}}}$ & $\Delta Z^{\mathrm{UB}, \min }$ & $\Delta Z^{\mathrm{UB}, \max }$ & $\sigma^{2}\left(\Delta Z^{\mathrm{UB}}\right)$ & $\overline{\mathrm{CPU}}{ }^{\mathrm{UB}}$ & $\mathrm{CPU}^{\mathrm{UB}, \min }$ & $\mathrm{CPU}^{\mathrm{UB}, \max }$ & $\sigma^{2}\left(\mathrm{CPU}^{\mathrm{UB}}\right)$ \\
\hline \multicolumn{9}{|l|}{ Set I: } \\
\hline $\operatorname{LPRH}(M 1)$ & 4.53 & 1.09 & 9.54 & 5.53 & 1.56 & 0.67 & 2.45 & 0.32 \\
\hline $\operatorname{LPRH}\left(M 1^{B}\right)$ & 4.53 & 1.09 & 9.54 & 5.53 & 1.56 & 0.67 & 2.45 & 0.33 \\
\hline $\operatorname{LPRH}\left(M 1^{A}\right)$ & 1.78 & 0.00 & 3.80 & 1.52 & 5.72 & 3.38 & 10.19 & 2.88 \\
\hline $\operatorname{LPRH}\left(M 1^{A, B}\right)$ & 1.78 & 0.00 & 3.80 & 1.52 & 5.70 & 3.39 & 10.29 & 2.91 \\
\hline LPRH $(M 2)$ & 5.51 & 1.34 & 15.87 & 11.93 & 12.78 & 7.95 & 17.39 & 6.54 \\
\hline $\operatorname{LPRH}\left(M 2^{B}\right)$ & 5.51 & 1.34 & 15.87 & 11.93 & 12.81 & 7.95 & 17.56 & 6.88 \\
\hline $\operatorname{LPRH}\left(M 2^{C}\right)$ & 1.30 & 0.00 & 7.36 & 3.35 & 184.50 & 78.76 & 362.29 & 6775.82 \\
\hline $\operatorname{LPRH}\left(M 2^{\mathrm{B}, \mathrm{C}}\right)$ & 1.30 & 0.00 & 7.36 & 3.35 & 184.15 & 79.00 & 361.42 & 6720.44 \\
\hline $\begin{array}{l}\text { SFLH } \\
\text { Set II: }\end{array}$ & 8.47 & 0.97 & 21.91 & 54.55 & 84.17 & 10.74 & 197.09 & 6511.45 \\
\hline $\operatorname{LPRH}(M 1)$ & 7.88 & 4.57 & 12.66 & 6.22 & 1.23 & 0.80 & 1.95 & 0.10 \\
\hline $\operatorname{LPRH}\left(M 1^{B}\right)$ & 7.88 & 4.57 & 12.66 & 6.22 & 1.22 & 0.76 & 2.04 & 0.11 \\
\hline $\operatorname{LPRH}\left(M 1^{A}\right)$ & 1.65 & 0.00 & 3.83 & 0.99 & 6.35 & 1.63 & 10.99 & 7.61 \\
\hline LPRH(M1 $\left.{ }^{A, B}\right)$ & 1.65 & 0.00 & 3.83 & 0.99 & 6.34 & 1.62 & 10.96 & 7.47 \\
\hline LPRH( $M 2)$ & 11.33 & 5.96 & 19.58 & 16.60 & 14.21 & 7.12 & 41.39 & 83.84 \\
\hline $\operatorname{LPRH}\left(M 2^{B}\right)$ & 11.33 & 5.96 & 19.58 & 16.60 & 14.28 & 7.04 & 41.65 & 86.75 \\
\hline LPRH $\left(M 2^{C}\right)$ & 1.52 & 0.00 & 6.59 & 2.90 & 211.12 & 177.05 & 306.53 & 1460.35 \\
\hline $\operatorname{LPRH}\left(M^{B, C}\right)$ & 1.52 & 0.00 & 6.59 & 2.90 & 211.04 & 176.74 & 307.46 & 1490.13 \\
\hline SFLH & 16.77 & 7.50 & 33.05 & 57.62 & 35.92 & 3.64 & 160.35 & 2101.85 \\
\hline
\end{tabular}

Table $3 \mathrm{c}$

Relation between capacity utilization and quality of the solutions for Set I

\begin{tabular}{|c|c|c|c|c|c|c|}
\hline \multirow[b]{3}{*}{ Relaxation } & \multicolumn{3}{|c|}{ Capacity utilization of plants } & \multicolumn{3}{|c|}{ Capacity utilization of WDUs } \\
\hline & $\rho=\mathrm{LOW}$ & $\rho=$ MEDIUM & $\rho=\mathrm{HIGH}$ & $\rho^{w}=$ LOW & $\rho^{w}=$ MEDIUM & $\rho^{w}=\mathrm{HIGH}$ \\
\hline & \multicolumn{6}{|l|}{$\Delta Z^{\mathrm{LB}}$} \\
\hline $\mathrm{LP}(M 1)$ & 9.92 & 8.39 & 5.53 & 11.05 & 6.81 & 5.98 \\
\hline $\operatorname{LP}\left(M 1^{B}\right)$ & 9.92 & 8.39 & 5.53 & 11.05 & 6.81 & 5.98 \\
\hline $\operatorname{LP}\left(M 1^{A}\right)$ & 2.52 & 2.91 & 3.32 & 9.04 & 8.64 & 7.72 \\
\hline $\operatorname{LP}\left(M 1^{A, B}\right)$ & 2.52 & 2.91 & 3.32 & 9.04 & 8.64 & 7.72 \\
\hline $\mathrm{LP}(M 2)$ & 9.92 & 8.39 & 5.53 & 11.05 & 6.81 & 5.98 \\
\hline $\mathrm{LP}\left(M 2^{B}\right)$ & 9.92 & 8.39 & 5.53 & 11.05 & 6.81 & 5.98 \\
\hline $\operatorname{LP}\left(M 2^{C}\right)$ & 0.28 & 0.35 & 0.46 & 0.42 & 0.20 & 0.47 \\
\hline $\operatorname{LP}\left(M 2^{B, C}\right)$ & 0.28 & 0.35 & 0.46 & 0.42 & 0.20 & 0.47 \\
\hline Heuristic & \multicolumn{6}{|l|}{$\overline{\Delta Z^{\bar{U}}}$} \\
\hline LPRH(M1) & 6.46 & 4.84 & 2.30 & 6.43 & 4.00 & 3.16 \\
\hline $\operatorname{LPRH}\left(M 1^{B}\right)$ & 6.46 & 4.84 & 2.30 & 6.43 & 4.00 & 3.16 \\
\hline $\operatorname{LPRH}\left(M 1^{A}\right)$ & 1.53 & 2.01 & 1.79 & 3.31 & 1.40 & 0.63 \\
\hline $\operatorname{LPRH}\left(M 1^{A, B}\right)$ & 1.53 & 2.01 & 1.79 & 3.31 & 1.40 & 0.63 \\
\hline LPRH $(M 2)$ & 7.89 & 5.60 & 3.03 & 8.31 & 4.48 & 3.73 \\
\hline $\operatorname{LPRH}\left(M 2^{B}\right)$ & 7.89 & 5.60 & 3.03 & 8.31 & 4.48 & 3.73 \\
\hline $\operatorname{LPRH}\left(M 2^{C}\right)$ & 1.03 & 1.41 & 1.46 & 2.30 & 0.96 & 0.64 \\
\hline $\operatorname{LPRH}\left(M 2^{B, C}\right)$ & 1.03 & 1.41 & 1.46 & 2.30 & 0.96 & 0.64 \\
\hline SFLH & 10.85 & 8.59 & 5.96 & 4.12 & 3.18 & 3.13 \\
\hline
\end{tabular}


Waste fractions $e_{i}$ are randomly generated from a uniform $U[0 ; 1]$ distribution. Variable transportation costs and demands are generated as in Set $I$.

\subsection{Computational results}

Below we discuss the performance of the lower bounding procedures and the upper bounding procedures with respect to their quality as well as the computational efforts required. The computational study was carried out on an IBM RISC System/6000 (Model 370) workstation.

\section{Lower bounding procedures}

The linear programming based lower bounding procedures were implemented in FORTRAN using the Optimization Subroutine Library OSL (IBM, 1990). ${ }^{2}$

In order to measure the quality of the lower bounding procedures we define for each problem instance $\alpha$ the (normalized) deviation between the value of the lower bound and the value of the optimal solution as

$\Delta Z_{\alpha}^{\mathrm{LB}}=\frac{Z_{\alpha}^{\mathrm{opt}}-Z_{\alpha}^{(\cdot)}}{Z_{\alpha}^{\mathrm{opt}}}$,

where $Z_{\alpha}^{\text {opt }}$ is the value of the optimal solution and $Z_{\alpha}^{(\cdot)}$ is the value corresponding to lower bounding procedure $(\cdot)$. Here, the optimal solution is obtained using the standard Branch and Bound procedure available in OSL (see also below). For each lower bounding procedure the average quality (aggregated over all instances in Set $\mathrm{I}$, respectively Set II) is denoted by $\overline{\Delta Z}^{\mathrm{LB}}$, the worst (best) case behaviour is denoted by $\Delta Z^{\mathrm{LB}, \max }$ $\left(\Delta Z^{\mathrm{LB}, \mathrm{min}}\right)$, and the variance is denoted by $\sigma^{2}\left(\Delta\left(Z^{\mathrm{LB}}\right)\right)$. Finally, $\overline{\mathrm{CPU}}$ is the average CPUtime (in seconds) over all problem instances within the set, CPU ${ }^{\min }\left(\mathrm{CPU}^{\max }\right.$ ) is the minimum (maximum) CPU time, and $\sigma^{2}$ (CPU) is the variance in CPU time. Table 3a provides an overview of the

\footnotetext{
${ }^{2}$ A preliminary study indicated that for our type of problems the primal simplex method with devex pricing outperforms interior point methods and dual simplex methods implemented in OSL.
}

above performance indicators for each of the lower bounding procedures. Table $3 \mathrm{c}$ provides some insights into the relationship between capacity utilization and the quality of the lower bounding procedures for instances from Set $I$.

From the computational results the following conclusions can be drawn:

- The CPU-time required to solve $\mathrm{LP}(M 1)$ is considerably less than the CPU-time required to solve LP $(M 2)$, whereas the quality of the solution does not differ between the two model formulations (Lemma 1),

- Adding valid inequalities $A$ to $\operatorname{LP}(M 1)$ and $C$ to $\operatorname{LP}(M 2)$ turns out to be very effective in terms of lower bound improvements for problem instances from Set I as well as from Set II. However, both inequality sets cause an increase in computational effort. Comparing set $\boldsymbol{A}$ with set $C$, we conclude that set $C$ contributes to larger bound improvements than set $A$ (Lemma 2 ), at the expense of a considerable increase in CPU-times (both in terms of averages and variances),

- Adding valid inequalities $B$ to $\operatorname{LP}(M 1)$ or LP(M2) does not seem to affect very much the quality of the solutions, nor the required computational effort (Lemma 3),

- From a comparison of the computational results for Set I and Set II it is concluded that the quality of the lower bounding procedures decreases when variability is introduced in capacities and fixed costs. Exceptions are the lower bounds $z_{\mathrm{LP}\left(M 2^{C}\right)}$ and $z_{\mathrm{LP}\left(M 2^{B C}\right)}$. The quality of these bounds seems to be rather insensitive to higher variabilities in capacities and fixed costs (the average deviation from optimality becomes even smaller for the two bounds). The influence of higher variability in capacities and fixed costs on CPU-times seems to be marginal, except for the bounds resulting from $\operatorname{LP}\left(M 2^{C}\right)$ and $\mathrm{LP}\left(M 2^{B C}\right)$,

- Furthermore, as shown in Table 3c capacities do influence the quality and required CPUtimes of the lower bounding procedures. It was discovered that higher capacitated problems (i.e. problems with higher $\rho$ and $\rho^{w}$ values) yield in general sharper lower bounds $z_{\operatorname{LP}(M 1)}$ and $Z_{\mathrm{LP}(M 2)}$. Comparing $z_{\mathrm{LP}(M 1)}$ with $z_{\mathrm{LP}\left(M 1^{A}\right)}$ we 
found that valid inequalities $\boldsymbol{A}$ become more effective in case of lower capacitated problems. This effect is explained by the observation that in this case the right hand side of constraints (8) and (9) becomes highly dependent on demand $d_{j}$, and maximum total waste $w_{i}$. These quantities act now as (surrogate) capacities, since they may dominate $s_{i}$ and $s_{k}^{w}$ (note that we use in fact the same arguments as in the formal proof of Lemma 2 ). The effectiveness of valid inequalities $\boldsymbol{C}$ does not seem to depend much on capacity utilization.

\section{Upper bounding procedures}

The results concerning the upper bounding procedures are shown in Table $3 \mathrm{~b}$ and in Table $3 \mathrm{c}$. Similarly to the presentation of the computational results for the lower bounding procedures, we define for each instance a the quality of upper bounding procedure $(\cdot)$ by

$$
\Delta Z^{\mathrm{UB}}=\frac{Z_{\alpha}^{(\cdot)}-Z_{\alpha}^{\mathrm{opt}}}{Z_{\alpha}^{\mathrm{opt}}} .
$$

Based on this performance indicator we compute over all instances in Set I respectively Set II the average quality for each upper bounding procedure $\left(\overline{\Delta Z}{ }^{\mathrm{UB}}\right)$, the worst case behaviour $\left(\Delta Z^{\mathrm{UB}, \max }\right)$, the best case behaviour $\left(\Delta Z^{\mathrm{UB}, \mathrm{min}}\right)$, the variance $\left(\sigma^{2}\left(\Delta\left(Z^{\mathrm{UB}}\right)\right)\right)$, and the average required CPU-time (CPU). Furthermore, CPU ${ }^{\text {min }}$ $\left(\mathrm{CPU}^{\mathrm{max}}\right.$ ) is the minimum (maximum) CPU time, and $\sigma^{2}(\mathrm{CPU})$ is the variance in CPU time.

With respect to the upper bounding procedures the following conciusions can be drawn:

- The solutions obtained by LPRH(M1) seems to be slightly better than the solutions obtained by LPRH $(M 2)$, although the latter procedure requires considerably more CPU-time. Though the difference in CPU-times was expected (see the discussion on lower bounds), we could not find intuitively appealing arguments to explain the small quality differences.

- Adding valid inequalities $A$ to $M 1$ or $C$ to $M 2$ yields better solutions to the round-off heuristics, both for Set I and Set II. This might be expected from the results reported for the lower bounding procedures, as better lower bounds have in general fewer setup variables with a value close to zero. Due to the latter, rounding-off a 'good' lower bounding solution yields in general better solutions than rounding-off a 'bad' lower bounding solution. By the same arguments it can be explained that (i) LPRH $\left(M 2^{C}\right)$ performs in general better than LPRH $\left(M 1^{A}\right)$, and (ii) the results to instances in Set $I$ are in general better than the results to instances in Set II, (iii) high capacitated problems yield on average better upper bounds than low capacitated problems Table (Table 3c),

- SFLH does not seem to perform very well when compared to the other procedures. Nevertheless, a preliminary study indicated that the quality of the results significantly improves when applying the multi-pass variant of SFLH (the quality of these solutions is comparable to the quality of the solutions to $M 1^{A}$ ). However, as indicated earlier, the multi-pass variant of SFLH very time consuming, and therefore not further investigated.

\section{Optimal solutions}

Optimal solutions - to which we compare the solutions of our upper bounding procedures were obtained using the standard Branch and Bound procedures available in OSL. It is remarkable that for many problem instances the optimal solutions to $M 1(M 2)$ could not be obtained within hours when valid inequalities $\boldsymbol{A}(C)$ where not added. However, when the valid inequalities were added very few branches were required to prove optimality, and the CPU time was reduced to less than 6 minutes at most (see Table 4). This demonstrates that valid inequalities $\boldsymbol{A}$ and $\boldsymbol{C}$ are essential when searching for optimal solutions.

Table 4

CPU-time to obtain optimal solutions

\begin{tabular}{lrrrr}
\hline & \multicolumn{5}{c}{ CPU-time in seconds } \\
\cline { 2 - 5 } Method & \multicolumn{1}{c}{ CPU } & \multicolumn{1}{c}{ CPU $^{\min }$} & CPU $^{\max }$ & $\sigma^{2}(\mathrm{CPU})$ \\
\hline Set I: & & & & \\
IP $\left(M 1^{A B}\right)$ & 36.92 & 4.58 & 177.99 & 2125.20 \\
IP $\left(M 2^{B C}\right)$ & 152.16 & 87.37 & 251.08 & 1580.41 \\
Set II: & & & & \\
IP $\left(M 1^{A B}\right)$ & 21.81 & 3.14 & 122.70 & 805.35 \\
IP $\left(M 2^{B C}\right)$ & 208.65 & 162.07 & 345.81 & 7147.27 \\
\hline
\end{tabular}




\section{Summary and conclusions}

In this paper we introduce the capacitated distribution and waste disposal problem. The problem is a variant of the two level capacitated facility location problem.

We formulate this NP-hard problem by two alternative mixed integer linear programs ( $M 1$ and $M 2$ ). We show that the LP-relaxations to both formulations yield the same lower bounding values. Also, for model $M 1(M 2)$ we derive valid inequality sets $\boldsymbol{A}$ and $\boldsymbol{B}$ ( $\boldsymbol{B}$ and $\boldsymbol{C}$ ). From a computational study we conclude that valid inequalities $\boldsymbol{A}$ and $\boldsymbol{C}$ are rather effective in strengthening the LP lower bound, although solving $\operatorname{LP}(M 2)$ with valid inequalities $C$ is rather time consuming. Furthermore, empirically we found that valid inequality set $B$ is not very effective in improving the LP lower bound. In addition to the LP based lower bounds we propose a Lagrangean relaxation procedure. Due to large CPU-times the procedure is not suitable for practical use. Summarizing, to generate good lower bounds against reasonable computational effort we advise to solve formulations $\mathrm{LP}(M 1)$ or $\mathrm{LP}(M 2)$ with valid inequalities $\boldsymbol{A}$ and $C$ respectively.

In addition to the lower bounding procedures we propose a number of upper bounding heuristics, based on (i) rounding-off the solutions to the LP relaxations, and (ii) constructing a feasible solution by sequentially solving two capacitated plant location problems. The effectiveness of the round-off strategies seems to depend heavily on the quality of the initial lower bounding solution. Therefore, the most effective round-off strategies exploit $\mathrm{LP}(M 1)$ or $\mathrm{LP}(M 2)$ with appropriate valid inequalities.

The sequential heuristic does not yield good results. The quality of the solutions obtained by the single pass version is poor. The multi-pass version yields solutions of reasonable quality, but is very expensive in terms of required CPU-time. Summarizing, we advise to use the LP based round-off strategies to obtain upper bounds. Using these upper bounds, optimal solutions can be obtained relatively fast, using a standard Branch and Bound procedure.
Finally, given the solution procedures that we have investigated in this paper, it can be concluded that: (i) Higher capacitated problems are in general easier to solve-than lower capacitated problems, and (ii) problems with higher variability in capacities and fixed costs are harder to solve than problems with lower variability.

\section{Acknowledgements}

The authors thank Monique Guignard and Choonho Ryu for making the computer code of the CAPLOC procedure available.

\section{Appendix}

Below we construct an example in which the solution to $\operatorname{LP}\left(M 2^{A}\right)$ is not feasible to $\operatorname{LP}\left(M 2^{C}\right)$. Consider the following problem instance with three customers, three plants, and two WDUs (dimensions $m=3 ; n=3 ; p=2$ ). Demand data are $d_{1}=d_{2}=d_{3}=1$. Fixed costs are $f_{1}=f_{2}=f_{3}$ $=3$, and $f_{1}^{w}=f_{2}^{w}=1$. Capacities of the plants are $s_{i}=2$ for $i=1,2,3$, and for the WDUs $s_{k}^{w}=3$ for $k=1,2$. Variables costs are $a_{13}=a_{21}$ $=a_{32}=3$, and $a_{i k}^{w}=1$ for all $i, k$. The other variable costs are zero. Finally, waste fractions equal one, i.e. $e_{1}=e_{2}=e_{3}=1 . \operatorname{LP}\left(M 2^{A}\right)$ yields an optimal solution with $z_{\mathrm{LP}\left(M 2^{A}\right)}=47 / 6$. The corresponding values for the location variables are: $Y_{1}=Y_{2}=Y_{3}=\frac{1}{2}$, and $Y_{1}^{w}=\frac{1}{3}, Y_{2}^{w}=0$. The flow variables have values $X_{11}=X_{12}=X_{22}=X_{23}$ $=X_{31}=X_{33}=\frac{1}{2}$, and $X_{11}^{w}=X_{21}^{w}=X_{31}^{w}=1$. The other flow variables are zero. By the definition of (a) and (b), it follows that the corresponding flow path variables equal $X_{111}=X_{121}=X_{221}=X_{231}=$ $X_{311}=X_{321}=\frac{1}{2}$. The other flow path variables are zero. This solution violates (19) for $k=1$.

\section{References}

Aardal, K. (1992), "On the solution of one and two-level capacitated facility location problems by the cutting plane approach", Ph.D. Thesis, Université Catholique de Louvain, Louvain-la-Neuve.

Christofides, N., and Beasley, J.E. (1983), "Extensions to a 
Lagrangean relaxation approach for the capacitated warehouse location problem", European Journal of Operational Research 12, 19-28.

Cornuejols, G., Sridharan, R., and Thizy, J.M. (1991), “A comparison of heuristics and relaxations for the capacitated plant location problem", European Journal of Operational Research 50, 280-297.

Davis, P.S., and Ray, T.L. (1969), "A branch and bound algorithm for the capacitated ties location problem", Naval Research Logistics Quarterly 16, 331-343.

Domschke, W., and Drexl, A. (1985), "ADD-heuristics starting procedures for capacitated location models", European Journal of Operational Research 21, 47-53.

Francis, R.L., McGinnis, L.F., and White, J.A. (1983), “Locational analysis", European Journal of Operational Research $12,220-252$.

Geoffrion, A.M. (1974), "Lagrangean relaxation for integer programming", Mathematical Programming 2, 82-114.

Guignard, M., and Opaswongkarn, K. (1990), "Lagrangean dual ascent algorithms for computing bounds in capacitated plant-location problems", European Journal of Operational Research 46, 73-83.
IBM Corporation (1991), Optimization Subroutine Library (OSL). Release 2. Guide and Reference, 3rd ed.

Khumawala, B.M. (1974), "An efficient heuristic procedure for the capacitated warehouse location problem", Naval Research Logistics Quarterly 21, 609-623.

Martello, S., and Toth, P. (1990) Knapsack Problems: Algo rithms and Computer Implementations, Wiley, New York.

Ro, H.-B., and Tcha, D.-W. (1984), "A branch and bound algorithm for the two-level uncapacitated facility location problem with some side constraints", European Journal of Operational Research 18, 349-358.

Bloemhof-Ruwaard, J.M., Salomon, M., and Van Wassenhove, L.N. (1994), "On coordination of product and byproduct flows in two-level distribution networks: Model formulations and solution procedures", European Journal of Operational Research 79/2, 325-339.

Ryu, C., and Guignard, M. (1992), "An efficient algorithm for the capacitated plant location problem", Working Paper. \#92-11-02, The Wharton School, Decision Sciences Department, University of Pennsyvlania. 